\title{
Comparison of local rosmarinic acid and topical dexpanthenol applications on wound healing in a rat experimental wound model
}

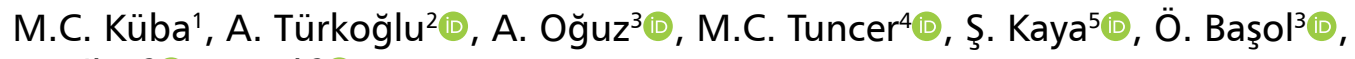 \\ H. Bilge ${ }^{3 \oplus}$, F. Tatı ${ }^{3(0)}$ \\ 'Department of General Surgery, Batman Regional Public Hospital, Batman, Turkey \\ ${ }^{2}$ Gaziantep Medical Park Hospital, Gaziantep, Turkey \\ ${ }^{3}$ Department of General Surgery, Faculty of Medicine, University of Dicle, Diyarbakır, Turkey \\ ${ }^{4}$ Department of Anatomy, Faculty of Medicine, University of Dicle, Diyarbakır, Turkey \\ ${ }^{5}$ Department of Pathology, Diyarbakır Educating and Research Hospital, Diyarbakır, Turkey
}

[Received: 8 June 2020; Accepted: 27 July 2020; Early publication date: 22 August 2020]

\begin{abstract}
Background: The aim of the study was to compare the effects of rosmarinic acid and dexpanthenol in a rat experimental wound model.

Materials and methods: Twenty-four Wistar albino rats weighing 200-250 g were randomly divided into three groups. After 2-cm full-thickness skin defects were created, the wounds were washed with sterile $0.9 \% \mathrm{NaCl}$ solution. After washing, the control group was left untreated, the second group received 5\% dexpanthenol cream, and the third group received 10\% rosmarinic acid cream. Before excision, the skin was evaluated macroscopically by measuring the reduction in wound size; after excision, histological examination (epithelisation, inflammation, fibrosis, granulation) was performed.

Results: Macroscopic comparison of the wound sizes showed that group 3 showed a statistically significant difference in wound size reduction compared to the other two groups. Histopathological examination showed that there was no statistically significant difference between the groups. We found that the rosmarinic acid group had greater wound size reduction than the other two groups. However, epithelialisation was detected in fewer cases.

Conclusions: We believe that rosmarinic acid can be used as a topical cream for wound healing, as it leads to significant reduction in wound size, resulting in fewer scars. (Folia Morphol 2021; 80, 3: 618-624)
\end{abstract}

Key words: wound healing, rosmarinic acid, dexpanthenol, rat

\section{INTRODUCTION}

Wound healing is a serious issue that may be associated with postoperative morbidity. Wound dehiscence and delayed wound healing remain important, serious problems in surgery. The basic principle of wound healing is to maintain adequate tissue perfu- sion and oxygenation, the anatomical and functional integrity of the affected area, and to ensure proper nutrition and moisture environment [17]. Various pharmacological agents have been studied for accelerating wound healing and preventing necrosis or ischaemia, and extensive efforts are still ongoing.

Address for correspondence: Prof. M.C. Tuncer, PhD, Dicle University, Medical School, Department of Anatomy, 21280, Diyarbakır, Turkey, tel: +90 4122488001 ext. 4539 (faculty room), fax: +90 412 2488440, mobile phone: +90 532 2744926, e-mail: drcudi@hotmail.com 
Sympatholytics, vasodilators, calcium channel blockers, anti-haemorrhagic agents, prostaglandin inhibitors, honey, anticoagulants, glucocorticoids, and free oxygen radical-inhibiting agents have been studied, and achieving various degrees of success. The most important disadvantages of many pharmacological agents are their relatively high doses and systemic use, which means that they have various potential adverse effects and risks. Local application, on the other hand, is more advantageous in terms of these risks $[1,6]$.

Dexpanthenol is widely used in wound healing in clinical practice. Pantothenate is a stimulant for migration, proliferation, and gene regulation in human dermal fibroblast cultures. Topical dexpanthenol is used both in wound care and for treating dermatological diseases because it stimulates skin regeneration and promotes wound healing [6].

Topical application of antioxidant-containing compounds is beneficial for wound healing and for protecting tissues from oxidative damage [8]. In chemical and cellular systems, rosmarinic acid (RA) and its basic metabolites have antioxidant activity [5]. RA also has anti-bacterial and nematicide effects and important anti-inflammatory properties $[5,7,10,21]$. As far as we know, the effect of RA on topical wound healing has not been investigated.

Dexpanthenol is widely used for small wounds and abrasions. Although dexpanthenol has been investigated widely for its effects on skin disorders, there has been insufficient evaluation of the effects of both RA and dexpanthenol on wound healing. In the present study, we evaluated the effects of topical RA and dexpanthenol on wound healing in a rat experimental wound model.

\section{MATERIALS AND METHODS}

This experimental study was submitted to the Ethics Committee of our University and approved by decision 2016/17 on 13 April, 2016. The experiments were performed in the University's Research and Application Centre Laboratory.

\section{Animals}

Twenty-four Wistar albino rats, each weighing an average of 200-250 g, were used. Starting 1 week before the study, the animals were prepared for the experiment, and were kept in a 12-h day/night environment in separate cages and given standard rat feed. The animals were randomly divided into three



Figure 1. A, B. Incision model created in rats.

groups, and fasted 12 hours before the experiment; they were allowed to drink only water.

\section{Skin defect model}

All animals were anesthetised by intramuscular administration of $50 \mathrm{mg} / \mathrm{kg}$ ketamine hydrochloride (Ketalar ${ }^{\circledast}$, Pfizer, Istanbul) and $5 \mathrm{mg} / \mathrm{kg}$ xylazine hydrochloride (Rompon ${ }^{\circledR}$, Bayer, Şişli, Istanbul) under aseptic conditions. The rats were anesthetised in the prone position, shaved, and povidone iodine was administered for antisepsis. A full-thickness skin defect $2 \mathrm{~cm}$ in length was created with a \#11 scalpel blade (Fig. 1). The wounds of all animals were cleaned with $0.9 \% \mathrm{NaCl}$ solution, and then the animals were divided into three groups.

Rosmarinic acid (96\% ALDRICH Chemistry Product, UK of United Kingdom) and dexpanthenol (Bepanthol ${ }^{\circledast}$, Bayer Turk Kimya San. Ltd. Sti. Istanbul) were used in the study. We used $18 \mathrm{~g}$ cold cream (cera alba, Olei Amygdalanum, Boracis, aqua rosae, oleum rosae) and $2 \mathrm{~g}$ powdered RA to prepare $10 \%$ RA cream.

Group 1: Control group. After receiving a full-thickness skin incision of approximately $2 \mathrm{~cm}$ on the dorsum, the wound was cleaned daily with isotonic saline solution, dressings were performed, and each rat was kept in a separate cage.

Group 2: Dexpanthenol group. After receiving a full-thickness skin incision of approximately $2-\mathrm{cm}$ diameter on the dorsum, the wound was cleaned with isotonic saline solution, and then dexpanthenol ( $5 \%$ cream) was applied daily; each rat was kept in a separate cage.

Group 3: Rosmarinic acid group. After receiving a full-thickness skin incision of approximately $2-\mathrm{cm}$ diameter on the dorsum, the wound was cleaned 
with isotonic saline solution, and then RA cream ( $10 \%$ cream) was applied daily; each rat was kept in a separate cage.

\section{Wound healing assessment}

The wound healing process was evaluated as follows: macroscopically, the reduction in wound size was calculated, and the excised wound tissue was evaluated by histological examination.

\section{Macroscopic evaluation}

Following the surgical procedure, the course of healing in all wounds was calculated using Walker's formula [20] after fixation of the rats' drawing on acetate paper on day $0,3,5,7,10,14$, and 21 . In addition, rats whose wound healing was completed were recorded during the daily control.

\section{Walker formula}

$$
\% \text { Wound area }=\frac{\text { Wound area on day } X}{\text { Wound area measured on day } 1} \times 100
$$

\section{Histological evaluation}

On day 21 , all animals were sacrificed, and $5 \times$ $\times 3 \mathrm{~cm}$ full-thickness skin, including the incision line, was removed from the dorsum for histological examination. A qualified pathologist evaluated the histopathological examinations. The tissues were fixed in $10 \%$ buffered formaldehyde solution for 2 days, and routine follow-up was performed. The tissues were embedded in paraffin blocks after the follow-up phase. Sections $(4 \mu \mathrm{m})$ obtained from the prepared paraffin blocks were stained with haematoxylin and eosin stain, examined under a light microscope, and photographed by a microscope-mounted camera. Inflammation, granulation tissue formation, and vascularisation were evaluated morphologically. Morphological findings, epithelialisation, cellular content (neutrophils, macrophages, fibroblasts), collagen regeneration, and vascularisation were scored as follows: $0-$ no change; 1 - little change; $2-$ moderate change; 3 - considerable change.

\section{Statistical analysis}

Statistical analysis was performed using SPSS for Windows 13.0 (SPSS Inc., Chicago, IL, USA). Categorical data were evaluated using the $\chi^{2}$ test. Continuous data were evaluated using the Kruskal-Wallis test, and the Mann-Whitney $U$ test was used for comparison of two groups. $\mathrm{P}<0.05$ was considered statistically significant.

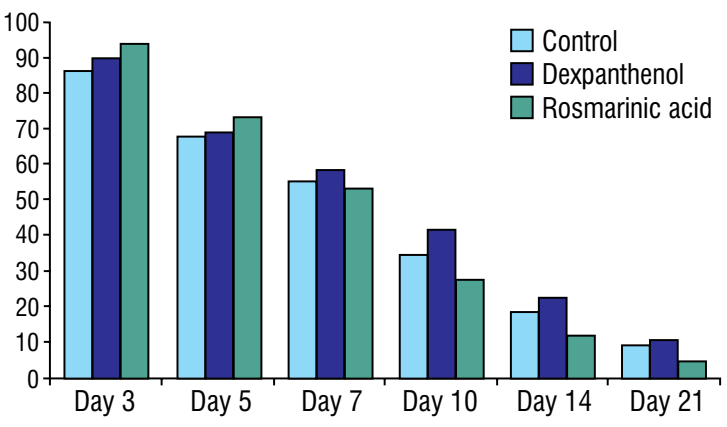

Figure 2. Reduction in wound size according to day.

\section{RESULTS}

\section{Evaluation of wound healing scores}

The wound healing scores calculated according to Walker's formula in the control, dexpanthenol, and RA groups are given in Figure 2 and Tables 1 and 2. The macroscopic and histopathological evaluations of the groups are as below.

\section{Macroscopic evaluation}

In postoperative day 3 , the greatest reduction in wound size was observed in the control group, but on day 10 and later, the greatest reduction in the wound size was observed in the RA group. The difference was statistically significant. However, no significant difference was observed in wound sizes between the groups on days 5 and 7 .

\section{Histopathological evaluation}

When the histopathological examination findings were compared statistically, no statistically significant difference was found between the groups in terms of epithelialisation, inflammation, fibrosis, and granulation (Figs. 3-5, Table 2).

\section{DISCUSSION}

Wound dehiscence and delayed wound healing are still important, serious problems in surgery. A proper wound healing process aims to improve the structure and function of the injured tissue. The healing process starts during an injury and can last for years $[7,10,20]$. Various clinicians use many agents topically and systemically for wound healing. Agents that are suggested to be useful in wound healing are reported to have antioxidant, antimicrobial, antibacterial, and anti-inflammatory properties $[11,13,18]$. Topical application of antioxidant-containing compounds for wound healing and for protecting tissues from oxidative damage has been shown to be beneficial [8]. 
Table 1. Distribution of wound healing score measurements.

\begin{tabular}{lcccccc}
\hline Group & \multicolumn{5}{c}{ Day-median (range) } \\
\cline { 2 - 7 } & $\mathbf{3}$ & $\mathbf{5}$ & $\mathbf{7}$ & $\mathbf{1 0}$ & $\mathbf{1 4}$ & $\mathbf{2 1}$ \\
\hline Control & $85(80-95)$ & $68(51-80)$ & $54.5(47-75)$ & $34(32-40)$ & $19.5(11-23)$ & $9(6-11)$ \\
Dexpanthenol & $89.5(85-93)$ & $70(65-72)$ & $59(51-64)$ & $40.5(37-50)$ & $22(17-28)$ & $9.5(7-14)$ \\
Rosmarinic acid & $94(89-96)$ & $72.5(66-83)$ & $52 .(44-64)$ & $30(16-36)$ & $11.5(9-17)$ & $4.5(3-7)$ \\
P-values (Kruskal-Wallis test) & 0.005903 & 0.397919 & 0.240973 & 0.000369 & 0.000743 & 0.000712 \\
\hline
\end{tabular}

Table 2. Comparison of histopathological evaluation results between groups

\begin{tabular}{llcccc}
\hline Parameters & & Control (\%) & Dexpanthenol (\%) & Rosmarinic acid (\%) & P \\
\hline Epithelisation & Present & $6(75 \%)$ & $5(62.5 \%)$ & $3(37.5 \%)$ & 0.1797 \\
Inflammation & Absent & $2(25 \%)$ & $3(37.5 \%)$ & $5(62.5 \%)$ & \\
& 0 & $1(12.5 \%)$ & $0(0 \%)$ & $0(0 \%)$ & 0.3302 \\
& 1 & $3(37.5 \%)$ & $3(37.5 \%)$ & $3(37.5 \%)$ & \\
Fibrosis & 2 & $3(37,5 \%)$ & $2(25 \%)$ & $5(62.5 \%)$ & \\
& 3 & $1(12.5 \%)$ & $3(37.5 \%)$ & $0(0 \%)$ & 0.4932 \\
& 0 & $0(0 \%)$ & $0(0 \%)$ & $0(0 \%)$ & \\
Granulation tissue & 1 & $1(12.5 \%)$ & $0(0 \%)$ & $0(0 \%)$ & $4(50 \%)$ \\
\end{tabular}

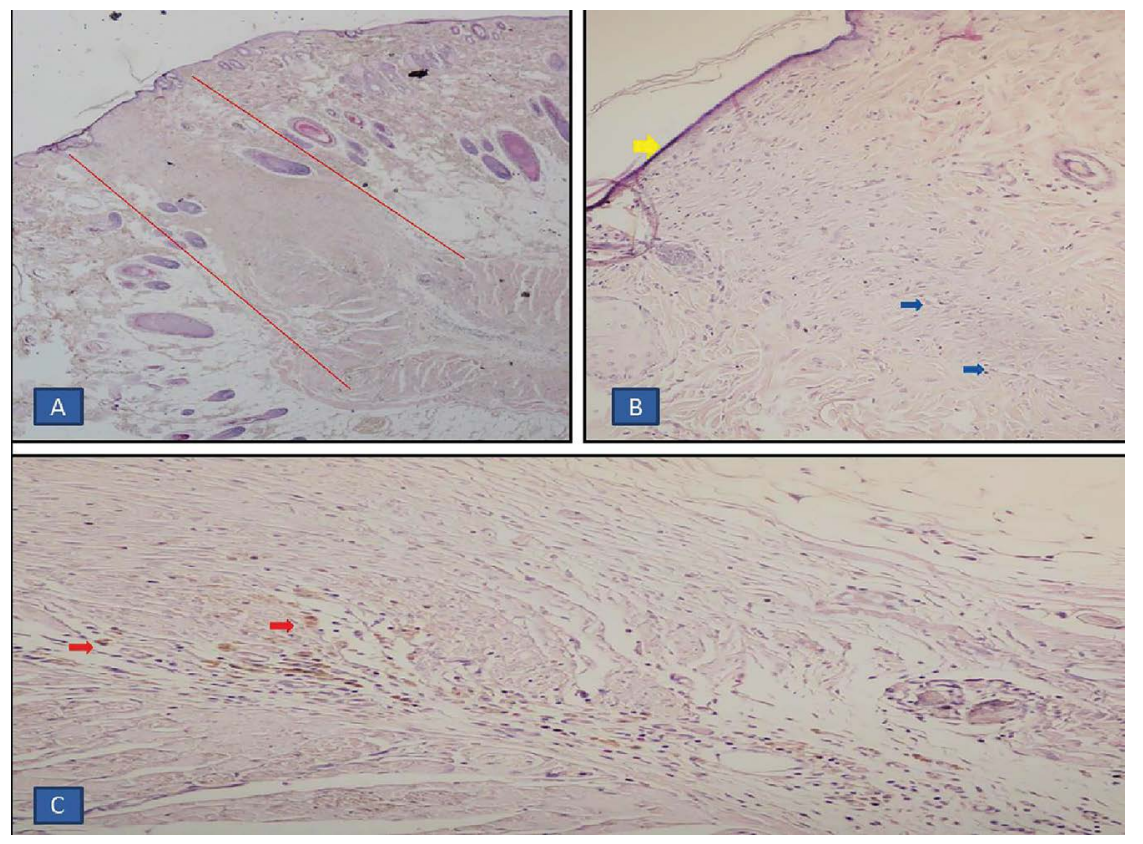

Figure 3. Microscopic view of wound tissues on day (control group). The area between the two red lines in the section belongs to the incision line (haematoxylin-eosin, $\times 40)(\mathbf{A})$. The yellow arrow indicates epithelialisation on the surface of the incision line; the blue arrows indicate the fibrotic line and vascular structures (haematoxylin-eosin, $\times 200$ ) (B). The red arrows indicate the pigmented macrophages of hemosiderin in brown at the bottom of the incision line (haematoxylin-eosin, $\times 200)$ (C). 


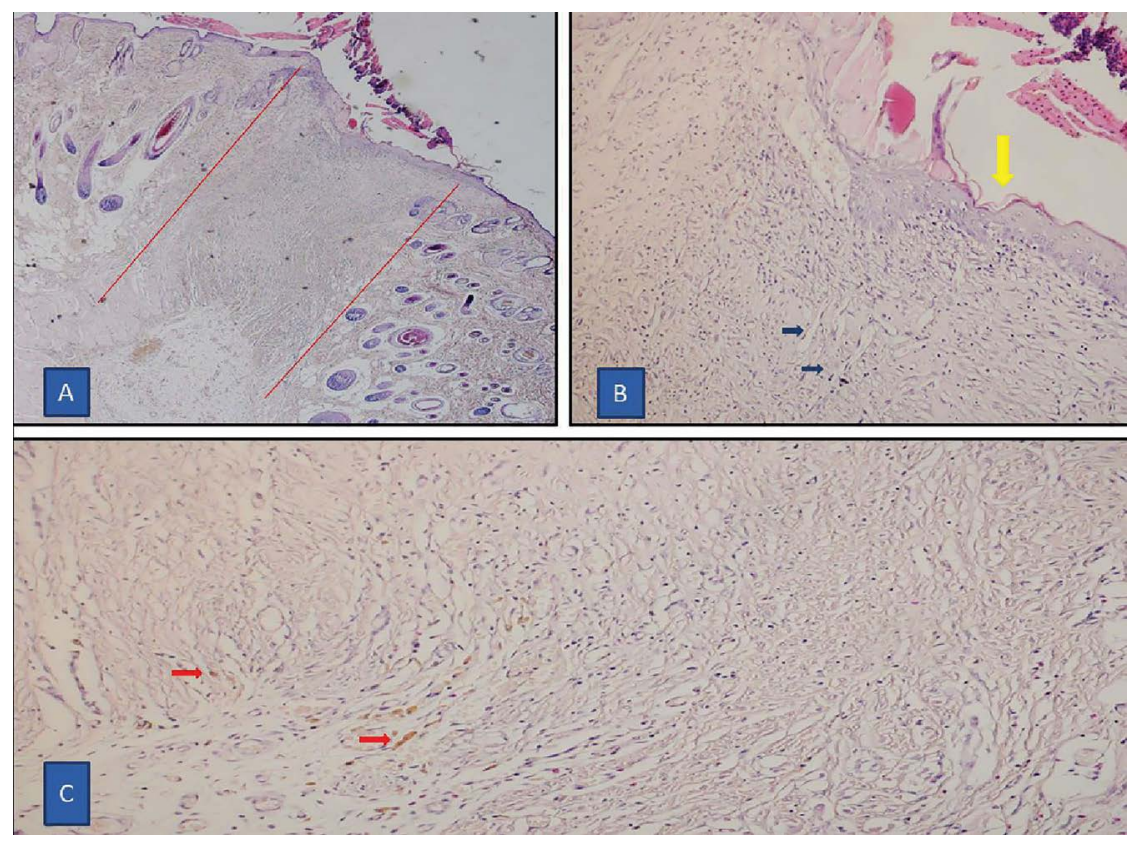

Figure 4. Microscopic view of wound tissues on day (dexpanthenol group). The area between the two red lines in the section belongs to the incision line (haematoxylin-eosin, $\times 40)(\mathbf{A})$. The yellow arrow indicates epithelialisation on the surface of the incision line; the blue arrows indicate the fibrotic line and vascular structures (haematoxylin-eosin, $\times 200$ ) (B). The red arrows indicate the pigmented macrophages of hemosiderin in brown at the bottom of the incision line (haematoxylin-eosin, $\times 200)$ (C).

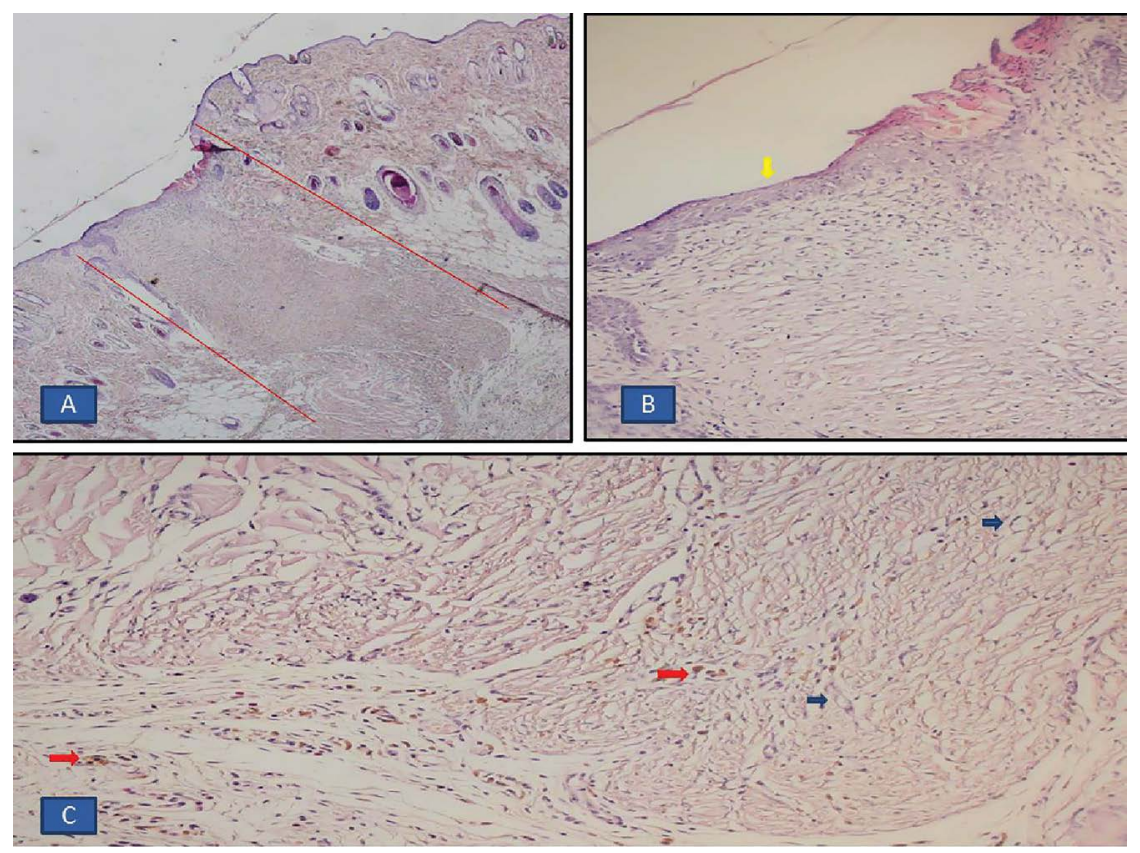

Figure 5. Microscopic view of wound tissues on day (rosmarinic acid group). The area between the two red lines in the section belongs to the incision line (haematoxylin-eosin, $\times 40)(\mathbf{A})$. The yellow arrow indicates epithelialisation on the surface in the incision line (haematoxylin-eosin, $\times 200)(B)$. The red arrows indicate the hemosiderin pigmented macrophages, and the blue arrows indicate the vascular structures at the bottom of the incision line (haematoxylin-eosin, $\times 200$ ) (C).

Mushtaq et al. [14] reported that RA has a protective effect against liver and kidney oxidative stress in diabetic rats. RA has an indirect antioxidant effect by affecting the production of cytoprotective genes in the liver, affecting the antioxidant system and nuclear factor-erythroid 2-related factor-2 (NRF2)-de- 
pendent phase II enzymes $[1,16]$. The antibacterial and anti-inflammatory effects of RA have also been demonstrated previously $[5,10,21]$.

In the present study, a significant reduction in wound size was detected in the RA group. On day 3, there was a statistically significant difference between the three groups in the reduction of wound area. This difference is probably due to the anti-inflammatory effect of RA. In early postoperative phase (day 3 ), the greatest reduction in wound size was observed in the control group, but in late postoperative phase (day 10 and later), the greatest reduction in wound size was observed in the RA group. The difference was statistically significant. However, no significant difference was observed in wound sizes between the groups on days 5 and 7 . However, histopathological examination did not reveal a significant difference in epithelisation, inflammation, fibrosis and granulation. Wound healing takes place in a multi-stage, multi-factorial mechanism. The reduction in wound size in favour of RA may be due to the effect of RA on fibroblast cells. Although RA reduced wound size more than dexpanthenol and the control group, the wound healing time was also partially prolonged in this group. This may be due to the blocking of the direct and indirect effects of mediators released from inflammatory cells due to the anti-inflammatory effect of RA. This may explain the inconsistency between the macroscopic findings and histomorphological findings.

Aramwit and Sangcakul [2] applied sericin cream and achieved $90 \%$ improvement on day 11 and achieved full recovery on day 15 . Kwon et al. [9] observed $90 \%$ improvement on day 10 and complete closure on day 14 with 14-day administration of topical recombinant human epidermal growth factor. In the present study, based on the significant statistical findings (approximately $90 \%$ on day 14 ; $>95 \%$ improvement on day 21 ), we concluded that the clinical effect of RA would be seen after day 7 and that RA should be applied for at least 3 weeks for maximum effect. Although the recovery times in our study appear slightly longer than that in the above studies, we believe that this may have been affected by the rat type, wound type, or other environmental factors used, as the recovery time in our control group was longer than that of their control groups.

Dexpanthenol is widely used in wound healing in clinical practice. Pantothenate is a stimulant for migration, proliferation, and gene regulation in hu- man dermal fibroblast cultures. Topical dexpanthenol is used both in wound care and in the treatment of dermatological diseases because it stimulates skin regeneration and promotes wound healing [6]. In a wound healing model, Ulger et al. [19] showed significantly better healing in the dexpanthenol and nebivolol groups than in the control group. Similarly, Oguz et al. [15] observed better recovery in the $\mathrm{N}$-acetylcysteine and dexpanthenol groups than in the control group. In our study, wound healing in the dexpanthenol group was similar to that of the control group, although RA group showed significantly better recovery than the dexpanthenol group. In addition, dexpanthenol caused more epithelialization than RA.

In the RA group, especially on day 7 and later, there was greater reduction in wound size compared to the other two groups. Histomorphological evaluation did not reveal a significant difference, but the evaluation of fibrosis showed that the number of RA rats with grade 3 fibrosis was higher than that of the other two groups. This finding partially supports the above results. Wound contraction is most active in the wound healing process between 7 and 10 days, when fibroblastic cell activation is also high. We believe that RA caused an increase in fibroblastic activity. More comprehensive studies are needed to demonstrate this precisely.

Our histopathological results do not statistically support our macroscopic observations. We did not find any significant difference in terms of inflammation between the groups. However, RA has known anti-inflammatory effects [4]. On the other hand, this effect is usually expressed through the levels of anti-inflammatory molecules. However, we did not analyse the level of pro-inflammatory molecules. Luo et al. [12] reported that RA had an anti-inflammatory effect on acute lung injury by decreasing the levels of pro-inflammatory molecules. Chen et al. [3] showed that RA ameliorated the fibrosis of pterygium epithelial cells by decreasing type I collagen production and downregulating transforming growth factor $\beta 1 /$ Smad signalling. However, we did not find a statistically significant effect of RA on fibrosis and fibroblast activity. This may indicate a need for new studies on the histopathological and anti-inflammatory effects of RA in a wound healing model. Unlike dexpanthenol, RA also has antibacterial and antiviral effects. These effects may lead RA being more effective than dexpanthenol in wound healing. 


\section{CONCLUSIONS}

In conclusion, there was greater reduction of wound size in the RA group compared to the dexpanthenol and control groups, but wound healing time was prolonged. In addition, epithelialisation was detected in fewer RA cases than in the other two groups. Significant reduction in wound size will result in less scarring during wound healing. Therefore, we believe that RA can be used in a topical cream for wound healing. However, additional experimental and clinical studies are needed for the duration and amount of use.

\section{Conflict of interest: None declared}

\section{REFERENCES}

1. Adomako-Bonsu AG, Chan SLf, Pratten M, et al. Antioxidant activity of rosmarinic acid and its principal metabolites in chemical and cellular systems: Importance of physico-chemical characteristics. Toxicol In Vitro. 2017; 40: 248-255, doi: 10.1016/j.tiv.2017.01.016, indexed in Pubmed: 28122265.

2. Aramwit $P$, Sangcakul $A$. The effects of sericin cream on wound healing in rats. Biosci Biotechnol Biochem. 2007; 71(10): 2473-2477, doi: 10.1271/bbb.70243, indexed in Pubmed: 17928707

3. Chen YY, Tsai CF, Tsai MC, et al. Anti-fibrotic effect of rosmarinic acid on inhibition of pterygium epithelial cells. Int J Ophthalmol. 2018; 11(2): 189-195, doi: 10.18240/ ijo.2018.02.02, indexed in Pubmed: 29487805.

4. Değer $U$, Çavuş $Y$. Investigation of the role of rosmarinic acid treatment in regulating inflammation, cell damage, and angiogenesis in rat ovarian torsion and detorsion models. Acta Cir Bras. 2020; 35(3): e202000304, doi: 10.1590/s0102-865020200030000004, indexed in Pubmed: 32692796.

5. Ekambaram SP, Perumal SS, Balakrishnan A, et al. Antibacterial synergy between rosmarinic acid and antibiotics against methicillin-resistant . J Intercult Ethnopharmacol. 2016; 5(4): 358-363, doi: 10.5455/jice.20160906035020, indexed in Pubmed: 27757265.

6. Heise R, Skazik C, Marquardt Y, et al. Dexpanthenol modulates gene expression in skin wound healing in vivo. Skin Pharmacol Physiol. 2012; 25(5): 241-248, doi: 10.1159/000341144, indexed in Pubmed: 22759998.

7. Jin BR, Chung KS, Cheon SY, et al. Rosmarinic acid suppresses colonic inflammation in dextran sulphate sodium (DSS)-induced mice via dual inhibition of NF- $\kappa$ B and STAT3 activation. Sci Rep. 2017; 7: 46252, doi: 10.1038/ srep46252, indexed in Pubmed: 28383063.

8. Kumar B, Vijayakumar M, Govindarajan R, et al. Ethnopharmacological approaches to wound healing--exploring medicinal plants of India. J Ethnopharmacol. 2007; 114(2): 103-113, doi: 10.1016/j.jep.2007.08.010, indexed in Pubmed: 17884316.
9. Kwon YB, Kim HW, Roh DH, et al. Topical application of epidermal growth factor accelerates wound healing by myofibroblast proliferation and collagen synthesis in rat. J Vet Sci. 2006; 7(2): 105-109, doi: 10.4142/jvs.2006.7.2.105, indexed in Pubmed: 16645332.

10. Liu JX, Zhang Y, Hu QP, et al. Anti-inflammatory effects of rosmarinic acid-4-O- $\beta$-D-glucoside in reducing acute lung injury in mice infected with influenza virus. Antiviral Res. 2017; 144: 34-43, doi: 10.1016/j.antiviral.2017.04.010, indexed in Pubmed: 28461072.

11. Liu M, Luo G, Wang $Y$, et al. Nano-silver-decorated microfibrous eggshell membrane: processing, cytotoxicity assessment and optimization, antibacterial activity and wound healing. Sci Rep. 2017; 7(1): 436, doi: 10.1038/ s41598-017-00594-x, indexed in Pubmed: 28348388.

12. Luo $C$, Zou L, Sun $H$, et al. A review of the anti-inflammatory effects of rosmarinic acid on inflammatory diseases. Front Pharmacol. 2020; 11: 153, doi: 10.3389/ fphar.2020.00153, indexed in Pubmed: 32184728.

13. Mulisa E, Asres K, Engidawork E. Evaluation of wound healing and anti-inflammatory activity of the rhizomes of Rumex abyssinicus J. (Polygonaceae) in mice. BMC Complement Altern Med. 2015; 15: 341, doi: 10.1186/ s12906-015-0878-y, indexed in Pubmed: 26423525.

14. Mushtaq N, Schmatz R, Ahmed M, et al. Protective effect of rosmarinic acid against oxidative stress biomarkers in liver and kidney of strepotozotocin-induced diabetic rats. J Physiol Biochem. 2015; 71(4): 743-751, doi: 10.1007/ s13105-015-0438-4, indexed in Pubmed: 26452500.

15. Oguz A, Uslukaya O, Alabalık U, et al. Topical N-acetylcysteine improves wound healing comparable to dexpanthenol: an experimental study. Int Surg. 2015; 100(4): 656-661, doi: 10.9738/INTSURG-D-14-00227.1, indexed in Pubmed: 25583306.

16. Oğuz A, Böyük A, Ekinci $A$, et al. Investigation of antioxidant effects of rosmarinic acid on liver, lung and kidney in rats: a biochemical and histopathological study. Folia Morphol. 2020; 79(2): 288-295, doi: 10.5603/FM.a2019.0087, indexed in Pubmed: 31436305.

17. Prisăcaru Al, Andrițoiu CV, Andriescu C, et al. Evaluation of the wound-healing effect of a novel Hypericum perforatum ointment in skin injury. Rom J Morphol Embryol. 2013; 54(4): 1053-1059, indexed in Pubmed: 24399001.

18. Qiu Z, Kwon AH, Kamiyama Y. Effects of plasma fibronectin on the healing of full-thickness skin wounds in streptozotocin-induced diabetic rats. J Surg Res. 2007; 138(1): 64-70, doi: 10.1016/j.jss.2006.06.034, indexed in Pubmed: 17161431.

19. Ulger BV, Kapan M, Uslukaya O, et al. Comparing the effects of nebivolol and dexpanthenol on wound healing: an experimental study. Int Wound J. 2016; 13(3): 367-371, doi: 10.1111/iwj.12314, indexed in Pubmed: 25040679.

20. Walker HL, Mason AD. A standard animal burn. J Trauma. 1968; 8(6): 1049-1051, doi: 10.1097/00005373196811000-00006, indexed in Pubmed: 5722120.

21. Wang J, Pan $X$, Han Yi, et al. Rosmarinic acid from eelgrass shows nematicidal and antibacterial activities against pine wood nematode and its carrying bacteria. Mar Drugs. 2012; 10(12): 2729-2740, doi: 10.3390/md10122729, indexed in Pubmed: 23201594. 